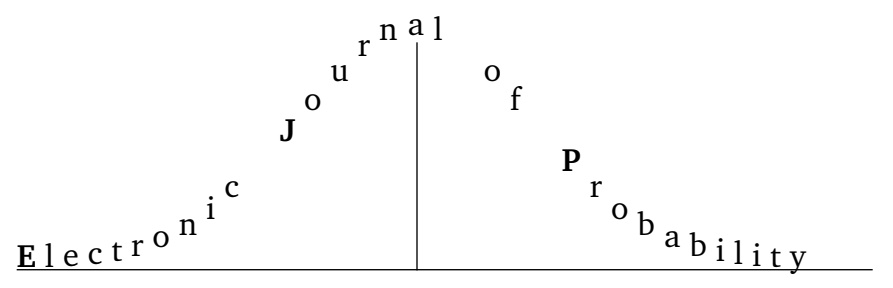

Vol. 16 (2011), Paper no. 29, pages 880-.

Journal URL

http://www.math.washington.edu/ ejpecp/

\title{
Approximation by the Dickman distribution and quasi-logarithmic combinatorial structures*
}

\author{
A. D. Barbour and Bruno Nietlispach \\ Institut für Mathematik, Universität Zürich \\ Winterthurertrasse 190, CH-8057 ZÜRICH \\ E-mails: a.d.barbour@math.uzh.ch; nietli@me.com
}

\begin{abstract}
Quasi-logarithmic combinatorial structures are a class of decomposable combinatorial structures which extend the logarithmic class considered by Arratia, Barbour and Tavaré (2003). In order to obtain asymptotic approximations to their component spectrum, it is necessary first to establish an approximation to the sum of an associated sequence of independent random variables in terms of the Dickman distribution. This in turn requires an argument that refines the Mineka coupling by incorporating a blocking construction, leading to exponentially sharper coupling rates for the sums in question. Applications include distributional limit theorems for the size of the largest component and for the vector of counts of the small components in a quasilogarithmic combinatorial structure.
\end{abstract}

Key words: Logarithmic combinatorial structures, Dickman's distribution, Mineka coupling .

AMS 2000 Subject Classification: Primary 60C05, 60F05, 05A16.

Submitted to EJP on August 4, 2010, final version accepted April 14, 2011.

\footnotetext{
*Work supported in part by Schweizerischer Nationalfonds Projekt Nr. 20-107935/1
} 


\section{Introduction}

Many of the classical random decomposable combinatorial structures, such as random permutations and random polynomials over a finite field, have component structure satisfying a conditioning relation: if $C_{i}^{(n)}$ denotes the number of components of size $i$, the distribution of the vector of component counts $\left(C_{1}^{(n)}, \ldots, C_{n}^{(n)}\right)$ of a structure of size $n$ can be expressed as

$$
\mathscr{L}\left(C_{1}^{(n)}, \ldots, C_{n}^{(n)}\right)=\mathscr{L}\left(Z_{1}, \ldots, Z_{n} \mid T_{0, n}=n\right),
$$

where $\left(Z_{i}, i \geq 1\right)$ is a fixed sequence of independent non-negative integer valued random variables, and $T_{a, n}:=\sum_{i=a+1}^{n} i Z_{i}, 0 \leq a<n$. If, as in the examples above, the $Z_{i}$ also satisfy

$$
i \mathbb{P}\left[Z_{i}=1\right] \rightarrow \theta \quad \text { and } \quad i \mathbb{E} Z_{i} \rightarrow \theta,
$$

the combinatorial structure is called logarithmic. It is shown in Arratia, Barbour and Tavaré (2003) $[\mathrm{ABT}]$ that combinatorial structures satisfying the conditioning relation and slight strengthenings of the logarithmic condition share many common properties. For instance, if $L^{(n)}$ is the size of the largest component, then $n^{-1} L^{(n)} \rightarrow_{d} L$, where $L$ has probability density function $f_{\theta}(x):=$ $e^{\gamma \theta} \Gamma(\theta+1) x^{\theta-2} p_{\theta}((1-x) / x), x \in(0,1]$, and $p_{\theta}$ is the density of the Dickman distribution $P_{\theta}$ with parameter $\theta$, given in Vervaat $\left(1972\right.$, p. 90). Furthermore, for any sequence $\left(a_{n}, n \geq 1\right)$ with $a_{n}=o(n)$,

$$
\lim _{n \rightarrow \infty} d_{\mathrm{TV}}\left(\mathscr{L}\left(C_{1}^{(n)}, \ldots, C_{a_{n}}^{(n)}\right), \mathscr{L}\left(Z_{1}, \ldots, Z_{a_{n}}\right)\right)=0 .
$$

Both of these convergence results can be complemented by estimates of the approximation error, under appropriate conditions. The asymptotic behaviour is quite different if $\mathbb{E} Z_{i} \sim i^{\alpha}$ for $\alpha \neq 1$ : see, for example, Freiman and Granovsky (2002) and Barbour and Granovsky (2005).

Knopfmacher (1979) introduced the notion of additive arithmetic semigroups, which give rise to decomposable combinatorial structures satisfying the conditioning relation, with negative binomially distributed $Z_{i}$. For these structures, $i \mathbb{P}\left[Z_{i}=1\right] \sim i \mathbb{E} Z_{i}=\theta_{i}$, where the $\theta_{i}$ do not always converge to a limit as $i \rightarrow \infty$. In those cases in which they do not, they become close to the integer skeleton of a sum of cosine functions with differing frequencies:

$$
\theta_{t}^{\prime}:=\theta+\sum_{l=1}^{L} \lambda_{l} \cos \left(2 \pi f_{l} t-\varphi_{l}\right), \quad t \in \mathbb{R},
$$

with $\sum_{l=1}^{L} \lambda_{l} \leq \theta$, and thus exhibit quasi-periodic behaviour. It is therefore natural to ask whether the asymptotic behaviour that holds generally for logarithmic combinatorial structures also holds for such structures, in which the $\theta_{i}$ do not converge, but certain averages of the $\theta_{i}$ do, and, if so, to find weaker forms of the logarithmic condition that are enough to imply the same asymptotic behaviour. In this paper, we define a family of combinatorial structures, the quasi-logarithmic class, that include the logarithmic structures as a special case, as well as those of Zhang (1996). For such structures, we give conditions under which $n^{-1} L^{(n)} \rightarrow_{d} L$ (Theorem 4.1) and $\lim _{n \rightarrow \infty} d_{\mathrm{TV}}\left(\mathscr{L}\left(C_{1}^{(n)}, \ldots, C_{a_{n}}^{(n)}\right), \mathscr{L}\left(Z_{1}, \ldots, Z_{a_{n}}\right)\right)=0$ (Theorem 4.3), just as in the logarithmic case. A key step in the proofs is to be able to show that, for sequences $a_{n}=\mathrm{o}(n)$, the normalized sum $n^{-1} T_{a_{n}, n}$ converges both in distribution and locally to the Dickman distribution $P_{\theta}$ (Theorems 
3.4 and 3.5), and that the error rates in these approximations can be controlled. To do so, it is in turn necessary to be able to show that, under suitable conditions,

$$
\lim _{n \rightarrow \infty} d_{\mathrm{TV}}\left(\mathscr{L}\left(T_{a_{n}, n}\right), \mathscr{L}\left(T_{a_{n}, n}+1\right)\right)=0, \quad \text { for all } a_{n}=\mathrm{o}(n),
$$

and that the error rate can be bounded by a power of $\left\{\left(a_{n}+1\right) / n\right\}$.

A number of the arguments used are adapted to the more general context from those presented in [ABT]. There, the sum $T_{0, n}:=\sum_{i=1}^{n} i Z_{i}$ is close in distribution to that of $T_{0, n}^{*}:=\sum_{i=1}^{n} i Z_{i}^{*}$, where $Z_{i}^{*} \sim \operatorname{Po}\left(\theta i^{-1}\right)$, and the latter sum has a compound Poisson distribution $\operatorname{CP}(\theta, n)$ whose properties are tractable. In the current situation, with the $\theta_{i}$ 's not all asymptotically equal, it is first necessary to show that $\operatorname{CP}(\theta, n)$ is still a good approximation to the sum $T_{0, n}$. This is by no means obviously the case. The intuition is nonetheless that, if the distributions of $T_{0, n}$ and $T_{0, n}+1$ are not too different, then having $\theta_{i}=2 \theta$ and $\theta_{i+1}=0$ instead of $\theta_{i}=\theta_{i+1}=\theta$ should leave the distribution $\mathscr{L}\left(T_{0, n}\right)$ more or less unchanged; only the average behaviour of the $\theta_{i}$ should be important. Thus we first want to establish (1.4). Once we have done so, we are able to show, by way of Stein's method, that $\mathscr{L}\left(T_{0, n}\right)$ is indeed close to $\mathrm{CP}(\theta, n)$

Proving that (1.4) holds under conditions appropriate for our quasi-logarithmic structures turns out in itself to be an interesting problem. The standard Mineka coupling, used to bound the total variation distance between a sum of independent, integer valued random variables and its unit translate, gives a very poor approximation in this context. To overcome the difficulty, we introduce a new coupling strategy, which yields a much more precise statement in a rather general setting (Theorem 2.1). This is the substance of the next section. We then show that the distributions $\mathscr{L}\left(T_{0, n}\right)$ and $\operatorname{CP}(\theta, n)$ are close in Section 3, and conclude that quasi-logarithmic combinatorial structures behave like logarithmic structures in Section 4.

As observed by Manstavičius (2009), when considering only the small components, the distances $d_{\mathrm{TV}}\left(\mathscr{L}\left(C_{1}^{(n)}, \ldots, C_{a_{n}}^{(n)}\right), \mathscr{L}\left(Z_{1}, \ldots, Z_{a_{n}}\right)\right)$ can be bounded, even without assuming that the $\theta_{j}$ 's converge on average to any fixed $\theta$, as long as they are bounded and bounded away from 0 (we do not require the latter condition). He considers only the case of Poisson distributed $Z_{i}$, for which, inspecting the proof of Theorem 4.3, it is enough to obtain an estimate of the form

$$
n\left|\mathbb{P}\left[T_{a_{n}, n}=n-k\right]-\mathbb{P}\left[T_{a_{n}, n}=n-l\right]\right| \leq C\{|k-l| / n\}^{\gamma}, \quad 0 \leq k, l \leq n / 2,
$$

for some $\gamma>0$. This he achieves by using his refined characteristic function arguments. Since we are also interested in approximating the distribution of the largest components, for which some form of convergence to a $\theta$ seems necessary, we do not attempt this refinement.

\section{An alternative to the Mineka coupling}

Let $\left\{X_{i}\right\}_{i \in \mathbb{N}}$ be mutually independent $\mathbb{Z}$-valued random variables, and let $S_{n}:=\sum_{i=1}^{n} X_{i}$. The Mineka coupling, developed independently by Mineka (1973) and Rösler (1977) (see also Lindvall (2002, Section II.14)) yields a bound of the form

$$
d_{\mathrm{TV}}\left(\mathscr{L}\left(S_{n}\right), \mathscr{L}\left(S_{n}+1\right)\right) \leq\left(\frac{\pi}{2} \sum_{i=1}^{n} u_{i}\right)^{-1 / 2}
$$

where

$$
u_{i}:=\left(1-d_{\mathrm{TV}}\left(\mathscr{L}\left(X_{i}\right), \mathscr{L}\left(X_{i}+1\right)\right)\right)
$$


see Mattner \& Roos (2007, Corollary 1.6). The proof is based on coupling copies $\left\{X_{i}^{\prime}\right\}_{i \in \mathbb{N}}$ and $\left\{X_{i}^{\prime \prime}\right\}_{i \in \mathbb{N}}$ of $\left\{X_{i}\right\}_{i \in \mathbb{N}}$ in such a way that

$$
V_{n}:=\sum_{i=1}^{n}\left(X_{i}^{\prime \prime}-X_{i}^{\prime}\right), \quad n \in \mathbb{N},
$$

is a symmetric random walk with steps in $\{-1,0,1\}$; the coupling inequality (Lindvall, 2002, Section I.2) then shows that

$$
d_{\mathrm{TV}}\left(\mathscr{L}\left(S_{n}\right), \mathscr{L}\left(S_{n}+1\right)\right) \leq \mathbb{P}[\tau>n]=\mathbb{P}\left[V_{n} \in\{-1,0\}\right],
$$

where $\tau$ is the time at which $\left\{V_{n}\right\}_{n \in \mathbb{Z}_{+}}$first hits level 1 , the last equality following from the reflection principle. However, this inequality gives slow convergence rates, if $X_{i}=i Z_{i}$ and the $Z_{i}$ are as described in the Introduction; typically, $d_{\mathrm{TV}}\left(\mathscr{L}\left(i Z_{i}\right), \mathscr{L}\left(i Z_{i}+1\right)\right)$ is extremely close to 1 , and, if $X_{i}$ is taken instead to be $(2 i-1) Z_{2 i-1}+2 i Z_{2 i}$, we still expect to have $1-d_{\mathrm{TV}}\left(\mathscr{L}\left(X_{i}\right), \mathscr{L}\left(X_{i}+1\right)\right) \asymp i^{-1}$, leading to bounds of the form

$$
d_{\mathrm{TV}}\left(\mathscr{L}\left(S_{n}\right), \mathscr{L}\left(S_{n}+1\right)\right)=\mathrm{O}\left((\log n)^{-1 / 2}\right) .
$$

In this section, by modifying the Mineka approach in the spirit of Rogers (1999) to allow the random walk $V$ to make larger jumps, we show that error bounds of order $n^{-\gamma}$ for some $\gamma>0$ can be achieved, representing an exponential improvement over the Mineka bounds.

Let $\left(X_{i}, i \geq 1\right)$ be independent $\mathbb{Z}_{+}$-valued random variables, set $S_{a, n}:=\sum_{i=a+1}^{n} X_{i}$, and define

$$
q(i, d):=\min \left\{\mathbb{P}\left[X_{i}=0\right] \mathbb{P}\left[X_{i+d}=i+d\right], \mathbb{P}\left[X_{i}=i\right] \mathbb{P}\left[X_{i+d}=0\right]\right\}, \quad i, d \in \mathbb{N} .
$$

Then it is possible to couple copies $\left(X_{i}^{\prime}, X_{i+d}^{\prime}\right)$ and $\left(X_{i}^{\prime \prime}, X_{i+d}^{\prime \prime}\right)$ of $\left(X_{i}, X_{i+d}\right)$ for any $i, d$ in such a way that

$$
\begin{aligned}
& \mathbb{P}\left[\left(X_{i}^{\prime}, X_{i+d}^{\prime}\right)=(0, i+d),\left(X_{i}^{\prime \prime}, X_{i+d}^{\prime \prime}\right)=(i, 0)\right] \\
& \quad=\mathbb{P}\left[\left(X_{i}^{\prime}, X_{i+d}^{\prime}\right)=(i, 0),\left(X_{i}^{\prime \prime}, X_{i+d}^{\prime \prime}\right)=(0, i+d)\right]=q(i, d) \\
& \mathbb{P}\left[\left(X_{i}^{\prime}, X_{i+d}^{\prime}\right)=\left(X_{i}^{\prime \prime}, X_{i+d}^{\prime \prime}\right)\right]=1-2 q(i, d) .
\end{aligned}
$$

Note that then

$$
\left(X_{i}^{\prime}+X_{i+d}^{\prime}\right)-\left(X_{i}^{\prime \prime}+X_{i+d}^{\prime \prime}\right)=\left\{\begin{array}{rc}
d & \text { with probability } q(i, d) ; \\
0 & \text { with probability } 1-2 q(i, d) ; \\
-d & \text { with probability } q(i, d),
\end{array}\right.
$$

so that sums of such differences, with non-overlapping indices, can be constructed so as to perform a symmetric random walk on $d \mathbb{Z}$. By successively coupling pairs in this way, and by using different values of $d$, it may thus be possible to couple the sums $S_{a, n}^{\prime}:=1+\sum_{i=a+1}^{n} X_{i}^{\prime}$ and $S_{a, n}^{\prime \prime}:=\sum_{i=a+1}^{n} X_{i}^{\prime \prime}$ quickly, even when many of the overlaps $q(i, d)$ are zero. The following theorem is typical of what can be achieved.

For $d \in \mathbb{N}$ and $\psi>0$, define $E(d, \psi):=\{i: q(i, d) \geq \psi /(i+d)\}$. For $D$ a subset of $\mathbb{N}$, suppose that there are $k \in \mathbb{N}$ and $\psi>0$ such that

$$
E(d, \psi) \cap\{j k+1, \ldots,(j+1) k\} \neq \emptyset, \quad \text { for all } d \in D, j \geq 1 .
$$


In particular, if $X_{i}=i Z_{i}$ with $Z_{i} \sim \operatorname{Po}\left(\theta_{i} / i\right)$, and if $0<\theta_{-} \leq \theta_{i}$ for all $i$, then clearly $q(i, d) \geq$ $\theta_{-} /(i+d)$ for all $i$ and $d \in \mathbb{N}$, and so (2.4) holds for any $D$ with $k=1$ and $\psi=\theta_{-}$. However, (2.4) also holds for any $D$ if, for instance, $0<\theta_{-} \leq \theta_{i}$ is only given for $i \in 3 \mathbb{N} \cup\{7 \mathbb{N}+2\}$, now with $k=21$ and $\psi=\theta_{-}$.

Theorem 2.1. Let $r, s \in \mathbb{N}$ be co-prime, and set

$$
D:=\{r\} \cup\left\{s 2^{g}, g \geq 0\right\} .
$$

Suppose that, for some $k, \psi$, (2.4) is satisfied with $D$ as above. Then there exist $C, \gamma>0$, depending on $r, s, k$ and $\psi$, such that

$$
d_{\mathrm{TV}}\left(\mathscr{L}\left(S_{a, n}\right), \mathscr{L}\left(S_{a, n}+1\right)\right) \leq 6\{(a+1) / n\}^{\gamma},
$$

for all $0 \leq a<n$ for which $a+1 \leq C n$.

Proof. We take $\widetilde{S}_{0}^{\prime}=1, \widetilde{S}_{0}^{\prime \prime}=0$, and then successively define $\widetilde{S}_{j}^{\prime}:=\widetilde{S}_{0}^{\prime}+\sum_{i \in I_{j}} X_{i}^{\prime}, \widetilde{S}_{j}^{\prime \prime}:=S_{0}^{\prime \prime}+\sum_{i \in I_{j}} X_{i}^{\prime \prime}$, $T_{j}:=\widetilde{S}_{j}^{\prime}-\widetilde{S}_{j}^{\prime \prime}, j \geq 1$. Here, the sequences $\left(X_{i}^{\prime}, 1 \leq i \leq n\right)$ and $\left(X_{i}^{\prime \prime}, 1 \leq i \leq n\right)$ are two copies of the sequence $\left(X_{i}, 1 \leq i \leq n\right)$ of independent random variables, constructed by successively coupling pairs $\left(X_{i_{j}}^{\prime}, X_{i_{j}+d_{j}}^{\prime}\right)$ and $\left(X_{i_{j}}^{\prime \prime}, X_{i_{j}+d_{j}}^{\prime \prime}\right)$, for suitable $i_{j}$ and $d_{j}$, realized independently of the random variables $\left(X_{i}^{\prime}, X_{i}^{\prime \prime}, i \in I_{j-1}\right)$, where $I_{j-1}:=\cup_{l=1}^{j-1}\left\{i_{l}, i_{l}+d_{l}\right\}$. This coupling of pairs typically omits some indices $i \in\{1,2, \ldots, n\}$; for such $i$, we set $X_{i}^{\prime}=X_{i}^{\prime \prime}$, chosen independently from $\mathscr{L}\left(X_{i}\right)$. The coupling of the pairs $\left(X_{i}^{\prime}, X_{i+d}^{\prime}\right)$ and $\left(X_{i}^{\prime \prime}, X_{i+d}^{\prime \prime}\right)$ is accomplished by arranging that $\mathscr{L}\left(\left(X_{i}^{\prime}, X_{i+d}^{\prime}\right)\right)=$ $\mathscr{L}\left(\left(X_{i}^{\prime \prime}, X_{i+d}^{\prime \prime}\right)\right)=\mathscr{L}\left(X_{i}\right) \times \mathscr{L}\left(X_{i+d}\right)$ and that $\left(X_{i}^{\prime}+X_{i+d}^{\prime}\right)-\left(X_{i}^{\prime \prime}+X_{i+d}^{\prime \prime}\right) \in\{-d, 0, d\}$, as described in $(2.2)$. The indices are defined by taking $i_{1}=\min \{i>a: i \in E(r, \psi)\}$, and then taking $i_{j+1}:=$ $\min \left\{i>i_{j}: i \in E\left(d_{j+1}, \psi\right), i, i+d_{j+1} \notin I_{j}\right\}$, where

$$
d_{l}:= \begin{cases}r, & \text { if } T_{l-1} \notin s \mathbb{Z} ; \\ s 2^{f_{2}\left(T_{l-1} / s\right)}, & \text { if } l>\tau, T_{l-1} \neq 0 ; \\ 0, & \text { if } T_{l-1}=0,\end{cases}
$$

and where $f_{2}(t)$ is the exponent of 2 in the prime factorization of $|t|, t \in \mathbb{Z}$. If $T_{l-1}=0$, we couple $X_{i_{l}}^{\prime}=X_{i_{l}}^{\prime \prime}$, and thus $X_{i_{l^{\prime}}}^{\prime}=X_{i_{l^{\prime}}}^{\prime \prime}$ for all $l^{\prime} \geq l$, with $i_{l^{\prime}}$ running through all $i>i_{l-1}$ such that $i \notin I_{l-1}$.

With this construction, the sequence $T_{j}$ can only change in jumps of size $\pm r$ until it first reaches $s \mathbb{Z}$. Thereafter, at any jump, the exponent $f_{2}\left(T_{j} / s\right)$ increases by 1 until $T_{j}$ is of the form $\pm s 2^{l}$ for some $l$; after this, the value of $T_{j}$ is either doubled or set to zero at each jump, in the latter case remaining in zero for ever. If $i_{J}+d_{J} \leq n$, where $J:=\inf \left\{j: T_{j}=0\right\}$, then

$$
S_{a, n}^{\prime}:=1+\sum_{i=a+1}^{n} X_{i}^{\prime}=\sum_{i=a+1}^{n} X_{i}^{\prime \prime}=: S_{a, n}^{\prime \prime},
$$

and $\mathscr{L}\left(S_{a, n}^{\prime}\right)=\mathscr{L}\left(S_{a, n}+1\right), \mathscr{L}\left(S_{a, n}^{\prime \prime}\right)=\mathscr{L}\left(S_{a, n}\right)$, so that, from the coupling inequality (Lindvall, 2002, Section I.2),

$$
d_{\mathrm{TV}}\left(\mathscr{L}\left(S_{a, n}\right), \mathscr{L}\left(S_{a, n}+1\right)\right) \leq \mathbb{P}\left[i_{J}+d_{J}>n\right] .
$$

We thus wish to bound this probability.

Now the process $T$, considered only at its jump times, has the law of a simple random walk of step length $r$ starting in 1, until it first hits a multiple of $s$, and the mean number of steps to do so is at 
most $s^{2} / 4$. Thus, and by the Markov property of the simple random walk, the number of jumps $N_{1}$ until a multiple of $s$ is hit is bounded in distribution by $\frac{1}{2} s^{2} G_{1}$, where $\mathbb{P}\left[G_{1}>j\right]=2^{-j}, j \geq 1$; in particular, for any $\gamma>0$,

$$
\mathbb{P}\left[N_{1}>\frac{1}{2} s^{2} \gamma \log _{2}\left(1 / \alpha_{n}\right)\right] \leq 2 \alpha_{n}^{\gamma},
$$

where $\alpha_{n}:=(a+1) / n$. The remaining number $N_{2}$ of jumps required for $T$ to reach 0 is then at most $\log _{2} r$ (in order to reach the form $\pm s 2^{l}$ for some $l$ ), together with an independent random number $G_{2}$ of steps until 0 is reached, having the same distribution as $G_{1}$; hence,

$$
\mathbb{P}\left[N_{2}>2 \gamma \log _{2}\left(1 / \alpha_{n}\right)\right] \leq 2 \alpha_{n}^{\gamma}
$$

also, if $n \geq(a+1) r^{1 / \gamma}$. It remains to show that the process $T$ has the opportunity to make this many jumps, with high probability, for suitable choice of $\gamma$.

Now, in view of (2.4), every block of indices $\{j k+1, \ldots,(j+1) k\}$ contains at least one $i \in E(r, \psi)$. Hence, for any $1 / 2 \leq \beta<1$, we can choose a set $S_{1}$ of non-overlapping pairs $\left(i_{l}, i_{l}+r\right), 1 \leq l \leq L$, such that $i_{l} \in E(r, \psi)$ and $a+1 \leq i_{l} \leq k(a+1) \alpha_{n}^{-\beta}-r$ for each $l$, and such that

$$
\sum_{l=1}^{L} \frac{1}{i_{l}+r}>\frac{1}{2(k \vee r)} \sum_{i=2}^{\left\lfloor(a+1) \alpha_{n}^{-\beta}\right\rfloor} \frac{1}{i+a /(k \vee r)} \geq \frac{\beta}{4(k \vee r)} \log \left(1 / \alpha_{n}\right),
$$

if $n \geq 3^{2 / \beta}(a+1)$. The first factor 2 in the denominator is present because a pair $(i, i+r)$ with $i \in$ $E(r, \psi)$ can be excluded from $S_{1}$, but only if $i=i_{l}+r$ for some pair $\left(i_{l}, i_{l}+r\right)$ already in $S_{1}$; the other is to yield an inequality, rather than an asymptotic equality. In similar fashion, for any nondecreasing sequence $\left(\rho_{l}, l \geq 1\right)$, we can choose a set $S_{2}$ of non-overlapping pairs $\left(i_{l}^{\prime}, i_{l}^{\prime}+s 2^{\rho_{l} \wedge l_{n}}\right)$, $1 \leq l \leq L^{\prime}$, where $l_{n}:=\left\lfloor\frac{1}{2} \log _{2} n\right\rfloor$, such that $k(a+1) \alpha_{n}^{-\beta}<i_{l}^{\prime} \leq n-s\lfloor\sqrt{n}\rfloor$ for each $l$, and such that

$$
\sum_{l=1}^{L^{\prime}} \frac{1}{i_{l}^{\prime}+s 2^{\rho_{l} \wedge l_{n}}}>\frac{1}{2 k} \sum_{i=\left\lceil 2(a+1) \alpha_{n}^{-\beta}\right\rceil+1}^{\lfloor n / k\rfloor} i^{-1} \geq \frac{1-\beta}{4 k} \log \left(1 / \alpha_{n}\right),
$$

if also $n \geq(a+1)(4 k)^{2 /(1-\beta)}$.

We now show that, for suitable choices of $\gamma$ and $\beta$, the pairs in $S_{1}$ with high probability yield $M_{1} \geq \frac{1}{2} s^{2} \gamma \log _{2} n$ jumps of $T$. We then show that those in $S_{2}$, with the sequence $\rho_{l}$ chosen in nonanticipating fashion such that $\rho_{1}$ is the exponent of 2 in $T_{l}$ at the first $l$ at which $T_{l} \in s \mathbb{Z}, \rho_{l+1}=\rho_{l}$ if $T_{l}=T_{l-1} \neq 0, \rho_{l+1}=\left(\rho_{l}+1\right) \wedge l_{n}$ if $0<T_{l} \neq T_{l-1}$ and $\rho_{l+1}=l_{n}$ otherwise, yield $M_{2} \geq 2 \gamma \log _{2} n$. Indeed, by the Chernoff inequalities (Chung \& Lu 2006, Theorem 3.1), if $\varphi_{1}, 0<\varphi_{1}<1$, is such that

$$
\frac{1}{2} s^{2} \gamma \log _{2}\left(1 / \alpha_{n}\right)=\frac{\beta \psi\left(1-\varphi_{1}\right)}{4(k \vee r)} \log \left(1 / \alpha_{n}\right),
$$

then

$$
\mathbb{P}\left[M_{1}<\frac{1}{2} s^{2} \gamma \log _{2}\left(1 / \alpha_{n}\right)\right] \leq \exp \left\{-3 \varphi_{1}^{2} \beta \psi \log \left(1 / \alpha_{n}\right) / 32(k \vee r)\right\} \leq \alpha_{n}^{\gamma},
$$

if $3 \varphi_{1}^{2} s^{2} /\left\{16\left(1-\varphi_{1}\right) \log 2\right\} \geq 1$. Similarly, using a martingale analogue of the Chernoff inequalities (Chung \& Lu 2006, Theorem 6.1), for $f_{2}$ such that $3 \varphi_{2}^{2} /\left\{4\left(1-\varphi_{2}\right) \log 2\right\} \geq 1$ and with

$$
2 \gamma \log _{2}\left(1 / \alpha_{n}\right)=\frac{(1-\beta) \psi\left(1-\varphi_{2}\right)}{4 k} \log \left(1 / \alpha_{n}\right)
$$


we get

$$
\mathbb{P}\left[M_{2}<2 \gamma \log _{2}\left(1 / \alpha_{n}\right)\right] \leq \exp \left\{-3 \varphi_{2}^{2}(1-\beta) \psi \log \left(1 / \alpha_{n}\right) / 32 k\right\} \leq \alpha_{n}^{\gamma} .
$$

Finally, for such choices of $f_{1}$ and $f_{2}$, equations (2.8) and (2.9) can be satisfed with the same choice of $\beta$ if $\gamma$ is chosen such that

$$
1=\frac{2 \gamma}{\psi \log 2}\left\{\frac{(k \vee r) s^{2}}{1-\varphi_{1}}+\frac{4 k}{1-\varphi_{2}}\right\}
$$

then

$$
\beta=\frac{2 \gamma(k \vee r) s^{2}}{\left(1-\varphi_{1}\right) \psi \log 2} .
$$

Choosing $\varphi_{2}$ to satisfy $3 \varphi_{2}^{2} /\left\{4\left(1-\varphi_{2}\right) \log 2\right\}=1$, and then $\varphi_{1}$ larger than its minimum value, if necessary, to ensure that $\beta \geq 1 / 2$, this yields the theorem.

Clearly, the exponent $\gamma$ could be sharpened; the condition (2.4) could also be weakened to one ensuring a positive density of indices in each $E(d, \psi)$ over longer intervals. The set $D$ could also be constructed in other ways. One natural extension would be to replace $r$ co-prime to $s$ with any $r_{1}, \ldots, r_{m}$ satisfying $\operatorname{gcd}\left\{r_{i}, \ldots, r_{m}, s\right\}=1$. Note also that if, for some $j_{0} \geq 1$,

$$
E(d, \psi) \cap\{j k+1, \ldots,(j+1) k\} \neq \emptyset, \quad \text { for all } d \in D, j \geq j_{0},
$$

then (2.4) holds with $k$ replaced by $j_{0} k$.

The coupling used to establish Theorem 2.1 is not the only possibility. In the example of additive arithmetic semigroups, there is one case in which the set $D$ can be taken to consist of the integers $\left\{2^{g+1}, g \geq 0\right\}$, but no odd integers. Here, the jumps in the process $T$ would always be even, and hence, since $T_{0}=1, T$ can never hit 0 . However, if we define

$$
\tilde{q}(i):=\min \left\{\mathbb{P}\left[X_{i}=0\right], \mathbb{P}\left[X_{i}=i\right]\right\} ; \quad \widetilde{E}(1, \psi):=\{i \in 2 \mathbb{Z}+1: \tilde{q}(i) \geq \psi / i\}
$$

and if, for all $j \geq 1$,

$$
\widetilde{E}(1, \psi) \cap\{j k+1, \ldots,(j+1) k\} \neq \emptyset,
$$

then one can begin the coupling construction by defining $X_{i}^{\prime}=X_{i}^{\prime \prime}$ for even $i$ and coupling $X_{i}^{\prime}$ and $X_{i}^{\prime \prime}$ for odd $i$ in such a way that

$$
\mathbb{P}\left[X_{i}^{\prime}=i, X_{i}^{\prime \prime}=0\right]=\mathbb{P}\left[X_{i}^{\prime}=0, X_{i}^{\prime \prime}=i\right]=1-\mathbb{P}\left[X_{i}^{\prime}=0, X_{i}^{\prime \prime}=0\right]=\tilde{q}(i),
$$

until the first time $i$ that $X_{i}^{\prime} \neq X_{i}^{\prime \prime}$, at which time the difference $T_{i}$ is even, taking either the value $i+1$ or $i-1$. Thereafter, the coupling is concluded using jumps of sizes $2^{g+1}$, with the second half of the strategy in the previous proof. Now the number of steps required to complete the coupling depends on how big the first even value of $T$ happens to be, but Chernoff bounds are still sufficient to be able to conclude the following theorem, which we state without proof.

Theorem 2.2. Suppose that, for some $k, \psi$, (2.4) is satisfied with $D=\left\{2^{g+1}, g \geq 0\right\}$, and (2.10) is also satisfied. Then there exist $C, \gamma>0$, depending on $k$ and $\psi$, such that

$$
d_{\mathrm{TV}}\left(\mathscr{L}\left(S_{a, n}\right), \mathscr{L}\left(S_{a, n}+1\right)\right) \leq C\{(a+1) / n\}^{\gamma},
$$

for all $0 \leq a<n$ 


\section{Approximation by the Dickman distribution}

As in the Introduction, let $\left(C_{1}^{(n)}, \ldots, C_{n}^{(n)}\right)$ be the component counts of a decomposable combinatorial structure of size $n$, related to the sequence of independent random variables $\left(Z_{i}, i \geq 1\right)$ through the Conditioning Relation (1.1). In this section, we wish to bound the distance between the distribution of the normalized sum $n^{-1} T_{a, n}:=n^{-1} \sum_{i=a+1}^{n} i Z_{i}$ and the Dickman distribution $P_{\theta}$, when the quantities $\theta_{i}:=i \mathbb{E} Z_{i}$ converge in some weak, average sense to $\theta$, and when $i \mathbb{P}\left[Z_{i}=1\right] \sim \theta_{i}$ also. In order to exploit the divisibility properties of the distributions of the random variables $Z_{i}$ that occur in many of the classical examples, it is convenient first to introduce some further notation.

As in $[\mathrm{ABT}]$, we suppose that the random variables $Z_{i}$ can be written as sums $Z_{i}:=\sum_{j=1}^{r_{i}} Z_{i j}$, where the random variables $\left(Z_{i j}, i \geq 1,1 \leq j \leq r_{i}\right)$ are all independent, and, for each $i$, the $Z_{i j}, 1 \leq j \leq r_{i}$ are identically distributed. This can always be taken to be the case, by setting $r_{i}=1$, but $r_{i}$ could be chosen arbitrarily large if $Z_{i}$ were infinitely divisible, and the bounds that we obtain may be smaller if the $r_{i}$ can be chosen to be large. We then define

$$
\varepsilon_{i k}:=\frac{i r_{i}}{\theta_{i}} \mathbb{P}\left[Z_{i 1}=k\right]-\mathbf{1}\{k=1\}, \quad k \geq 1,
$$

so that $\sum_{k \geq 1} k \varepsilon_{i k}=0$ for all $i$, since $\theta_{i}=i \mathbb{E} Z_{i}$, and the $\varepsilon_{i k}$ can be expected to be small if also $i \mathbb{P}\left[Z_{i}=1\right] \sim \theta_{i}$. Finally, we set $\mu_{i}:=\sum_{k \geq 1} k \sup _{j \geq i}\left|\varepsilon_{j k}\right|$, which we assume to be finite.

We now specify our analogue of $(1.2)$. Clearly, assuming $\mu_{i} \rightarrow 0$ yields random variables $Z_{i}$ that mostly only take the values 0 or 1 , but we also need some regularity among the $\theta_{i}$. To make this precise, we define

$$
\delta(m, \theta):=\sup _{j \geq 0}\left|\theta-\frac{1}{m} \sum_{i=1}^{m} \theta_{j m+i}\right|
$$

and assume that it converges to zero, for some $\theta>0$, as $m \rightarrow \infty$. Note that this already implies that

$$
\theta_{\text {sup }}:=\sup _{i \geq 1} \theta_{i}<\infty
$$

In addition, we need to be able to apply Theorem 2.1, with $Z_{i}$ for $X_{i}$ and with $T_{a, n}$ for $S_{a, n}$. For this, we need practical conditions on the $\theta_{i}$ which imply that (2.4) is satisfied for $D$ as in (2.5), for some $r, s, k$ and $\psi$. So we define $i_{0}:=\min \left\{i \geq 3 \theta_{\text {sup }}: \mu_{i} \leq 1 / 2\right\}$, and set $E^{\prime}(d, \psi):=\{i \geq$ $\left.i_{0}: \min \left(\theta_{i}, \theta_{i+d}\right) \geq \psi\right\}$. We now show that $E^{\prime}(d, \psi) \subset E(d, \psi / 8) \cap\left[i_{0}, \infty\right)$, so that, if (2.4) is satisfied with $E^{\prime}$ for $E$, for some $r, s$ coprime, $\psi>0$ and $D$ defined in (2.5), then the conditions of Theorem 2.1 are satisfied with the same values of $r, s$ and $k$, and with $\psi / 8$ for $\psi$.

Lemma 3.1. Suppose that $i \geq i_{0}$ and that $\min \left(\theta_{i}, \theta_{i+d}\right) \geq \psi$. Then

$$
q(i, d):=\min \left\{\mathbb{P}\left[Z_{i}=0\right] \mathbb{P}\left[Z_{i+d}=i+d\right], \mathbb{P}\left[Z_{i}=i\right] \mathbb{P}\left[Z_{i+d}=0\right]\right\} \geq \psi / 8(i+d) .
$$

Proof. Note first that, for $i \geq i_{0}$,

$$
\mathbb{P}\left[Z_{i 1}=1\right]=\frac{\theta_{i}}{i r_{i}}\left(1+\varepsilon_{i 1}\right) \geq \frac{\theta_{i}}{2 i r_{i}} ; \quad \mathbb{P}\left[Z_{i 1} \geq 1\right]=\frac{\theta_{i}}{i r_{i}}\left(1+\sum_{k \geq 1} \varepsilon_{i k}\right) \leq \frac{3 \theta_{i}}{2 i r_{i}},
$$

so that

$$
\mathbb{P}\left[Z_{i}=0\right]=\left(1-\mathbb{P}\left[Z_{i} \geq 1\right]\right)^{r_{i}} \geq\left(1-\frac{3 \theta_{i}}{2 i r_{i}}\right)^{r_{i}} \geq\left(1-\frac{3 \theta_{i}}{2 i}\right) \geq \frac{1}{2} ;
$$


thus also

$$
\mathbb{P}\left[Z_{i}=1\right] \geq \frac{\theta_{i}}{2 i} \mathbb{P}\left[Z_{i}=0\right] \geq \frac{\theta_{i}}{4 i}
$$

and the lemma follows.

Using these preparations, we can now state relatively straightforward conditions under which a decomposable combinatorial structure can be said to be quasi-logarithmic. Our simplest condition is the following.

Definition 3.2. We say that a decomposable combinatorial structure satisfies the quasi-logarithmic condition QLC if it satisfies the Conditioning Relation (1.1), if

$$
\lim _{i \rightarrow \infty} \mu_{i}=0 ; \quad \lim _{m \rightarrow \infty} \delta(m, \theta)=0 \text { for some } \theta>0 \text {, }
$$

and if, for some $r, s$ coprime, $\psi>0$ and $D$ defined in (2.5), (2.4) is satisfied with $E^{\prime}$ for $E$.

For quantitative estimates, a slightly stronger assumption is useful.

Definition 3.3. We say that a decomposable combinatorial structure satisfies the quasi-logarithmic condition QLC2 if it satisfies the Conditioning Relation (1.1), if

$$
\mu_{i}=O\left(i^{-\alpha}\right) ; \quad \delta(m, \theta)=O\left(m^{-\beta}\right) \text { for some } \theta, \alpha, \beta>0
$$

and if, for some $r, s$ coprime, $\psi>0$ and $D$ defined in (2.5), (2.4) is satisfied with $E^{\prime}$ for $E$.

Under such conditions, we now prove the close link between $\mathscr{L}\left(n^{-1} T_{a, n}\right)$ and $P_{\theta}$. Our method of proof involves showing first that $\mathscr{L}\left(T_{0, n}\right)$ is close to the compound Poisson distribution $\operatorname{CP}(\theta, n):=$ $\mathscr{L}\left(\sum_{i=1}^{n} i Z_{i}^{*}\right)$, where the $Z_{i}^{*} \sim \operatorname{Po}\left(i^{-1} \theta\right)$ are independent; the closeness of $n^{-1} \mathrm{CP}(\theta, n)$ and $P_{\theta}$ is already known [ABT, Theorems 11.10 and 12.11], and the Wasserstein distance between $\mathscr{L}\left(n^{-1} T_{0, n}\right)$ and $\mathscr{L}\left(n^{-1} T_{a, n}\right)$ is at most $n^{-1} \sum_{i=1}^{a} \theta_{i}$.

To bound the distance between $\mathscr{L}\left(T_{a, n}\right)$ and $\operatorname{CP}(\theta, n)$, we use Stein's method (Barbour, Chen \& Loh 1992). For any Lipschitz test function $f: \mathbb{Z}_{+} \rightarrow \mathbb{R}$, one expresses $f$ in the form

$$
f(j)-\operatorname{CP}(\theta, n)\{f\}=\theta \sum_{i=1}^{n} g_{f}(j+i)-j g_{f}(j),
$$

for an appropriate function $g_{f}$ [ABT, Chapter 9.1]. Hence, for instance, the Wasserstein distance between $\mathscr{L}\left(T_{a, n}\right)$ and $\operatorname{CP}(\theta, n)$ can be estimated by bounding

$$
\begin{aligned}
& \left|\mathbb{E}\left\{\theta \sum_{i=1}^{n} g_{f}\left(T_{a, n}+i\right)-T_{a, n} g_{f}\left(T_{a, n}\right)\right\}\right| \\
& =\left|\sum_{i=a+1}^{n} \mathbb{E}\left\{\theta g_{f}\left(T_{a, n}+i\right)-i Z_{i} g_{f}\left(T_{a, n}\right)\right\}+\sum_{i=1}^{a} \theta \mathbb{E} g_{f}\left(T_{a, n}+i\right)\right|,
\end{aligned}
$$

uniformly for Lipschitz functions $f \in \mathrm{Lip}_{1}$, for which functions $\left\|g_{f}\right\| \leq 1$ [ABT, (9.14)]. The right hand side can now be relatively easily bounded. 
First, we re-express the element

$$
\mathbb{E}\left\{i Z_{i} g_{f}\left(T_{a, n}\right)\right\}=\sum_{l=1}^{r_{i}} \mathbb{E}\left\{i Z_{i l} g_{f}\left(T_{a, n}\right)\right\}
$$

of (3.5) by observing that

$$
\mathbb{E}\left\{i Z_{i l} g_{f}\left(T_{a, n}\right)\right\}=\frac{\theta_{i}}{r_{i}}\left\{\mathbb{E} g_{f}\left(T_{a, n}^{(i)}+i\right)+\sum_{k \geq 1} k \varepsilon_{i k} \mathbb{E} g_{f}\left(T_{a, n}^{(i)}+i k\right)\right\},
$$

where $T_{a, n}^{(i)}:=T_{a, n}-i Z_{i 1}, a<i \leq n$. Hence, to bound (3.5), we have

$$
\begin{aligned}
\mid \mathbb{E}\{\theta & \left.\sum_{i=a+1}^{n} g_{f}\left(T_{a, n}+i\right)-T_{a, n} g_{f}\left(T_{a, n}\right)\right\} \mid \\
\leq & \left|\sum_{i=a+1}^{n}\left\{\left(\theta-\theta_{i}\right) \mathbb{E} g_{f}\left(T_{a, n}+i\right)+\theta_{i} \mathbb{E}\left[g_{f}\left(T_{a, n}+i\right)-g_{f}\left(T_{a, n}^{(i)}+i\right)\right]\right\}\right| \\
& \quad+\sum_{i=a+1}^{n} \theta_{i} \sum_{k \geq 1} k\left|\varepsilon_{i k}\right| \mathbb{E}\left|g_{f}\left(T_{a, n}^{(i)}+i k\right)\right|,
\end{aligned}
$$

and

$$
\begin{aligned}
& \mathbb{E}\left\{g_{f}\left(T_{a, n}+i\right)-g_{f}\left(T_{a, n}^{(i)}+i\right)\right\} \\
& =\frac{\theta_{i}}{i r_{i}}\left\{\mathbb{E}\left\{g_{f}\left(T_{a, n}^{(i)}+2 i\right)-g_{f}\left(T_{a, n}^{(i)}+i\right)\right\}\right. \\
& \left.\quad+\sum_{k \geq 1} \varepsilon_{i k} \mathbb{E}\left\{g_{f}\left(T_{a, n}^{(i)}+i(k+1)\right)-g_{f}\left(T_{a, n}^{(i)}+i\right)\right\}\right\} ;
\end{aligned}
$$

and, clearly,

$$
\theta \sum_{i=1}^{a}\left|\mathbb{E} g_{f}\left(T_{a, n}+i\right)\right| \leq a \theta\left\|g_{f}\right\|
$$

With the help of these estimates, we can prove the following approximation theorem. We use the notation $D^{1}(T)$ to denote $d_{\mathrm{TV}}(\mathscr{L}(T), \mathscr{L}(T+1)) ; D^{1}\left(T_{a, n}\right)$ appears in the bound (3.9) by way of (3.11), and is controlled by applying Theorem 2.1 with $Z_{i}$ for $X_{i}$.

Theorem 3.4. With the definitions above,

$$
d_{\mathrm{w}}\left(\mathscr{L}\left(n^{-1} T_{a, n}\right), \mathrm{P}_{\theta}\right) \leq n^{-1}(1+\theta)^{2}+\min _{1 \leq m \leq n} \varepsilon_{1}(n, a, m)
$$

where $\varepsilon_{1}(n, a, m)$ is given in (3.11). If QLC holds, $d_{\mathrm{w}}\left(\mathscr{L}\left(n^{-1} T_{a_{n}, n}\right), \mathrm{P}_{\theta}\right) \rightarrow 0$ for any sequence $a_{n}=$ $o(n)$. If QLC2 holds, then $d_{\mathrm{w}}\left(\mathscr{L}\left(n^{-1} T_{a, n}\right), \mathrm{P}_{\theta}\right)=O\left(\{(a+1) / n\}^{\eta_{1}}\right)$ for some $\eta_{1}>0$. 
Proof. We first consider $d_{\mathrm{w}}\left(\mathscr{L}\left(T_{a, n}\right), \mathrm{CP}(\theta, n)\right)$, for which we bound the quantities appearing in (3.5), as addressed in (3.6)-(3.8). The contribution from (3.8) is immediate. Then, defining

$$
\theta^{*}:=\max \left\{1, \theta, \sup _{i \geq 1} \theta_{i}\right\} \quad \text { and } \quad \sigma_{n}^{*}:=\sum_{i=1}^{n} \max \left\{\mu_{i}, \frac{1}{i r_{i}}\right\},
$$

we can easily bound the third element in (3.6) by $\theta^{*} \sigma_{n}^{*}\left\|g_{f}\right\|$, and the second, using (3.7), contributes at most $4 \theta^{*} \sigma_{n}^{*}\left\|g_{f}\right\|$, since also $i r_{i} \geq 1$. For the first term, we use Lemma 5.2(i) to give

$$
\left|\sum_{i=1}^{n}\left(\theta-\theta_{i}\right) \mathbb{E} g_{f}\left(T_{a, n}+i\right)\right| \leq\left\{2 \theta^{*} m+n \delta(m, \theta)+(1 / 4) \theta^{*} m n D^{1}\left(T_{a, n}\right)\right\}\left\|g_{f}\right\| .
$$

In all, and using $\left\|g_{f}\right\| \leq 1$, this gives the bound

$$
d_{\mathrm{w}}\left(\mathscr{L}\left(T_{a, n}\right), \operatorname{CP}(\theta, n)\right) \leq n \varepsilon_{1}(n, a, m)
$$

with

$$
\varepsilon_{1}(n, a, m):=\frac{1}{4} \theta^{*} m D^{1}\left(T_{a, n}\right)+\delta(m, \theta)+n^{-1} \theta^{*}\left\{5 \theta^{*} \sigma_{n}^{*}+2 m+a\right\} .
$$

This bound, together with the inequality

$$
d_{\mathrm{w}}\left(\mathscr{L}\left(n^{-1} T_{a, n}\right), \mathrm{P}_{\theta}\right) \leq n^{-1} d_{\mathrm{w}}\left(\mathscr{L}\left(T_{a, n}\right), \mathrm{CP}(\theta, n)\right)+d_{\mathrm{w}}\left(n^{-1} \mathrm{CP}(\theta, n), \mathrm{P}_{\theta}\right),
$$

now give the required estimate, since

$$
d_{\mathrm{w}}\left(n^{-1} \mathrm{CP}(\theta, n), \mathrm{P}_{\theta}\right) \leq n^{-1}(1+\theta)^{2}
$$

see [ABT, Theorem 11.10].

If QLC holds, $D^{1}\left(T_{a, n}\right)=O\left(\{(a+1) / n\}^{\gamma}\right)$ for some $\gamma>0$, and choosing $m=m_{n}$ tending to infinity slowly enough ensures that $\varepsilon_{1}\left(n, a_{n}, m_{n}\right) \rightarrow 0$. If QLC2 holds, choose $m$ to be an appropriate power of $\{(a+1) / n\}$.

With a little more difficulty, one can prove the analogous local approximation to the distribution of $T_{a, n}$. This is the main tool for establishing the asymptotic behaviour of quasi-logarithmic combinatorial structures.

Theorem 3.5. For any $0 \leq a \leq n$ and any $r \geq 2 a+1$, we have

$$
\left|n \mathbb{P}\left[T_{a, n}=r\right]-p_{\theta}(r / n)\right| \leq \min _{1 \leq m \leq n} \varepsilon_{5}(n, a, m ; r),
$$

with $\varepsilon_{5}(n, a, m ; r)$ as defined in (3.24) below. If QLC holds, it follows that $\sup _{r \geq n x}\left|n \mathbb{P}\left[T_{a, n}=r\right]-p_{\theta}(r / n)\right| \rightarrow 0$ for any $x>0$ and any sequence $a_{n}=o(n)$. If QLC2 holds, then $\sup _{r \geq n x}\left|n \mathbb{P}\left[T_{a, n}=r\right]-p_{\theta}(r / n)\right|=O\left(\{(a+1) / n\}^{\eta_{2}}\right)$, for any $x>0$ and for some $\eta_{2}>0$. 
Proof. With $x:=r / n$, we begin by writing

$$
\begin{aligned}
\left|p_{\theta}(x)-n \mathbb{P}\left[T_{a, n}=r\right]\right| \leq & \frac{1}{x}\left|\theta \mathbb{P}\left[r-n \leq T_{a, n}<r-a\right]-r \mathbb{P}\left[T_{a, n}=r\right]\right| \\
& +\left|p_{\theta}(x)-\frac{1}{x} \theta \mathbb{P}\left[r-n \leq T_{a, n}<r-a\right]\right| .
\end{aligned}
$$

Now the quantity

$$
\Delta_{1}(r):=\theta \mathbb{P}\left[r-n \leq T_{a, n}<r-a\right]-r \mathbb{P}\left[T_{a, n}=r\right]
$$

is of the form $\mathbb{E}\left\{\theta \sum_{i=a+1}^{n} g\left(T_{a, n}+i\right)-T_{a, n} g\left(T_{a, n}\right)\right\}$, as in 3.6, with $g:=\mathbf{1}_{\{r\}}$. Take $l_{0}$ such that $\mathbb{P}\left[Z_{l 1}=0\right] \geq 1 / 2$ for all $l \geq l_{0}$, and set $C\left(l_{0}\right):=\left\{\min _{1 \leq l \leq l_{0}} \max _{j \geq 1} \mathbb{P}\left[Z_{l 1}=j\right]\right\}^{-1}$; then we have

$$
\mathbb{P}\left[T_{a, n}^{(i)}=s\right] \leq 2 \mathbb{P}\left[T_{a, n}=s\right], i \geq l_{0} ; \quad \mathbb{P}\left[T_{a, n}^{(i)}=s\right] \leq C\left(l_{0}\right) \sup _{j \geq 1} \mathbb{P}\left[T_{a, n}=j\right],
$$

for all $s \geq 0$ and $1 \leq i<l_{0}$. Note also that, by considering expectations of functions of the form $\mathbf{1}_{[0, j]}$,

$$
\sup _{j \geq 1} \mathbb{P}\left[T_{a, n}=j\right] \leq D^{1}\left(T_{a, n}\right) .
$$

Using these bounds, we can bound the third element in 3.6 by

$$
\theta^{*}\left\{\sum_{i=1}^{l_{0}-1} C\left(l_{0}\right) \mu_{i} D^{1}\left(T_{a, n}\right)+\sum_{i=l_{0}}^{l-1} 2 \mu_{i} D^{1}\left(T_{a, n}\right)+2 \mu_{l}\right\},
$$

for any $l \geq l_{0}$, since $\sum_{i=l}^{n} \mathbb{P}\left[T_{a, n}=r-i k\right] \leq 1$ for all $k \geq 1$. The second element is bounded, using (3.7), in a very similar way, giving

$$
2 \theta^{*}\left\{\sum_{i=1}^{l-1}\left(C\left(l_{0}\right) \vee 2\right) \frac{1}{i r_{i}}\left(1+\mu_{i}\right) D^{1}\left(T_{a, n}\right)+\frac{2}{l r_{l}}\left(1+\mu_{l}\right)\right\} .
$$

Finally, the first element in $(3.6)$ is bounded by Lemma 5.2(ii) as

$$
\left|\sum_{i=a+1}^{n}\left(\theta-\theta_{i}\right) \mathbb{P}\left[T_{a, n}=r-i\right]\right| \leq \delta(m, \theta)+m \theta^{*}\left(2+\frac{m}{6}\right) D^{1}\left(T_{a, n}\right) .
$$

Combining these estimates, we conclude that, for any $l \geq l_{0}$,

$$
\left|\Delta_{1}(r)\right| \leq \varepsilon_{2}(n, a, m)
$$

where

$$
\begin{aligned}
& \varepsilon_{2}(n, a, m):= \\
& \theta^{*} \min _{l \geq l_{0}}\left\{\sum_{i=1}^{l-1}\left(C\left(l_{0}\right) \vee 2\right)\left(\frac{2}{i r_{i}}\left(1+\mu_{i}\right)+\mu_{i}\right) D^{1}\left(T_{a, n}\right)+4\left(\frac{1}{l r_{l}}\left(1+\mu_{l}\right)+\mu_{l}\right)\right\} \\
&+m \theta^{*}\left(2+\frac{m}{6}\right) D^{1}\left(T_{a, n}\right)+\delta(m, \theta) .
\end{aligned}
$$


The next step is to bound the difference

$$
\Delta_{2}(r):=\mathbb{P}\left[r-n \leq T_{a, n}<r-a\right]-\mathrm{CP}(\theta, n)\{[r-n, r-a-1]\},
$$

which can once again be accomplished by using (3.4) and (3.5). Since, for $f:=\mathbf{1}_{[0, s-1]}$,

$$
\left\|g_{f}\right\| \leq(1+\theta) /(s+\theta)
$$

by [ABT, Lemma 9.3], it follows as in the proof of (3.10) in the previous theorem that

$$
\left|\mathbb{P}\left[T_{a, n}<s\right]-\operatorname{CP}(\theta, n)\{[0, s-1]\}\right| \leq s^{-1}(1+\theta) n \varepsilon_{1}(n, a, m),
$$

for any $s \geq 1$. For $2 a<r \leq n$, this gives

$$
\left|\Delta_{2}(r)\right| \leq(r-a)^{-1}(1+\theta) n \varepsilon_{1}(n, a, m) \leq 2 r^{-1} n(1+\theta) \varepsilon_{1}(n, a, m) .
$$

For $r>n$, two differences as in (3.17) are needed. The first is just as before; the second is bounded by

$$
\varepsilon_{3}(n, a, m ; r):=\min \left\{(r-n)^{-1}(1+\theta) n \varepsilon_{1}(n, a, m), \mathbb{P}\left[T_{a, n}<r-n\right]+\mathrm{CP}(\theta, n)\{[0, r-n-1]\}\right\},
$$

where the alternative is useful if $r$ is close to $n$. Now

$$
\mathrm{CP}(\theta, n)\{[0, j]\} \leq \prod_{i=j+1}^{n} \operatorname{Po}\left(\theta i^{-1}\right)\{0\}=\exp \left\{-\sum_{i=j+1}^{n} \theta i^{-1}\right\} \leq\left(\frac{j+1}{n+1}\right)^{\theta} .
$$

Rather similarly,

$$
\begin{aligned}
& \mathbb{P}\left[T_{a, n} \leq j\right] \leq \prod_{i=j+1}^{n}\left\{\mathbb{P}\left[Z_{i 1}=0\right\}^{r_{i}} \leq \exp \left\{-\sum_{i=j+1}^{n} \theta_{i} i^{-1}\left(1-\mu_{i}\right)\right\}\right. \\
& \quad \leq\left[\exp \left\{-\sum_{i=j+1}^{n}\left(\frac{\theta_{i}-\theta}{i}\right)\right\}\left(\frac{j+1}{n+1}\right)^{\theta}\right]^{1-\mu_{j+1}} \leq\left\{2 e^{1+\theta^{*}}\left(\frac{j+1}{n+1}\right)^{\theta-\delta(j / 2, \theta)}\right\}^{1-\mu_{j+1}},
\end{aligned}
$$

from Lemma 5.3 , and this in turn gives

$$
\mathbb{P}\left[T_{a, n} \leq j\right] \leq 2 e^{1+\theta^{*}}\left(\frac{\left(j \vee j_{0}\right)+1}{n+1}\right)^{\theta / 4},
$$

where $\mu_{j+1} \leq 1 / 2$ and $\delta(j / 2, \theta) \leq \theta / 2$ for all $j \geq j_{0}$. Using (3.19) and 3.20) in (3.18), and optimizing with respect to $r$, gives

$$
\varepsilon_{3}(n, a, m ; r) \leq 4 e^{1+\theta^{*}}\left\{\varepsilon_{1}(n, a, m)\right\}^{\theta /(4+\theta)}=: \varepsilon_{4}(n, a, m) .
$$

Hence

$$
\left|\Delta_{2}(r)\right| \leq 2 n r^{-1}(1+\theta) \varepsilon_{1}(n, a, m)+\varepsilon_{4}(n, a, m)
$$


for all $r \geq 2 a+1$.

The remainder of the estimate is concerned with comparing the density $p_{\theta}(r / n)$ with $n r^{-1} \theta \mathrm{CP}(\theta, n)\{[r-n, r-a-1]\}$. From [ABT, Theorem 11.12], it follows that

$$
\left|\mathrm{CP}(\theta, n)\{[r-n, r-a-1]\}-P_{\theta}\{[r / n-1,(r-a) / n)\}\right| \leq c(\theta) n^{-(\theta \wedge 1)},
$$

for a constant $c(\theta)$, and then, from [ABT, (4.23) and (4.20)],

$$
\begin{aligned}
& \left|n r^{-1} \theta P_{\theta}\{[r / n-1,(r-a) / n)\}-p_{\theta}(r / n)\right| \\
& \quad=n r^{-1} \theta\left|P_{\theta}\{[r / n-1,(r-a) / n)\}-P_{\theta}\{[r / n-1, r / n)\}\right| \leq c^{\prime}(\theta) n r^{-1}(a / n)^{(\theta \wedge 1)}
\end{aligned}
$$

so long as $r \geq 2 a$. Combining (3.15), (3.21), (3.22) and (3.23), the theorem follows with

$$
\begin{aligned}
& \varepsilon_{5}(n, a, m ; r):=\frac{n}{r}\left\{2 \theta(1+\theta) n r^{-1} \varepsilon_{1}(n, a, m)+\varepsilon_{2}(n, a, m)\right. \\
&\left.+\theta \varepsilon_{4}(n, a, m)+c^{\prime \prime}(\theta)((a+1) / n)^{(\theta \wedge 1)}\right\} ;
\end{aligned}
$$

note that, for $2 a \leq n / 2 \leq r \leq n$, the bound can be replaced by the uniform

$$
\varepsilon_{5}^{\prime}(n, a, m):=2\left\{\varepsilon_{2}(n, a, m)+4 n^{-1} \theta(1+\theta) \varepsilon_{1}(n, a, m)+c^{\prime \prime}(\theta)((a+1) / n)^{(\theta \wedge 1)}\right\} .
$$

If QLC holds, $D^{1}\left(T_{a, n}\right)=O\left(\{(a+1) / n\}^{\gamma}\right)$ for some $\gamma>0$, and choosing $m=m_{n}$ tending to infinity slowly enough ensures that $\varepsilon_{l}\left(n, a_{n}, m_{n}\right) \rightarrow 0$ for $l=1,2$ and 4; this implies that $\varepsilon_{5}\left(n, a_{n}, m_{n}, r\right) \rightarrow 0$ uniformly in $r \geq n x$, for any $x>0$. If QLC2 holds, choose $m$ to be an appropriate power of $\{(a+1) / n\}$.

\section{Quasi-logarithmic structures}

In this section, we consider the two common properties shared by logarithmic combinatorial structures that were discussed in the Introduction, and show that they are also true for quasi-logarithmic structures. For each of the properties, the local approximation of $\mathbb{P}\left[T_{a, n}=r\right]$ in Theorem 3.5 is the fundamental relation from which everything else follows. Other aspects of the asymptotic behaviour of logarithmic combinatorial structures could be extended to quasi-logarithmic structures by analogous methods.

\subsection{The size of the largest component}

The following theorem is an extension of a result proved by Kingman (1977) in the case of $\theta$-tilted random permutations. A version for logarithmic structures can be found in [ABT, Theorem 7.13].

Theorem 4.1. Let

$$
L^{(n)}:=\max \left\{1 \leq i \leq n: C_{i}^{(n)}>0\right\}
$$

be the size of the largest component. Then, if QLC holds,

$$
\lim _{n \rightarrow \infty} \mathscr{L}\left(n^{-1} L^{(n)}\right)=\mathscr{L}(L)
$$


where $L$ is a random variable concentrated on $(0,1]$, whose distribution is given by the density function

$$
f_{\theta}(x):=e^{\gamma \theta} \Gamma(\theta+1) x^{\theta-2} p_{\theta}((1-x) / x), \quad \text { for all } x \in(0,1] .
$$

In particular, if $\theta=1$,

$$
\lim _{n \rightarrow \infty} \mathbb{P}\left[L^{(n)} \leq n / y\right]=\rho(y), \quad \text { for all } y \geq 1,
$$

where $\rho$ is Dickman's function (Dickman, 1930).

Proof. Fix $x \in(0,1]$. Then

$$
\mathbb{P}\left[n^{-1} L^{(n)} \leq x\right]=\mathbb{P}\left[C_{\lfloor n x\rfloor+1}^{(n)}=\cdots=C_{n}^{(n)}=0\right]=\prod_{i=\lfloor n x\rfloor+1}^{n} \mathbb{P}\left[Z_{i}=0\right] \frac{\mathbb{P}\left[T_{0,\lfloor n x\rfloor}=n\right]}{\mathbb{P}\left[T_{0, n}=n\right]} .
$$

Theorem 3.5yields

$$
\frac{\mathbb{P}\left[T_{0,\lfloor n x\rfloor}=n\right]}{\mathbb{P}\left[T_{0, n}=n\right]}=\frac{n p_{\theta}(n /\lfloor n x\rfloor)}{\lfloor n x\rfloor p_{\theta}(1)}\left\{1+O\left(\min _{m}\left\{\varepsilon_{5}(\lfloor n x\rfloor, 0, m ; n)\right\}+\min _{m}\left\{\varepsilon_{5}(n, 0, m ; n)\right\}\right)\right\} .
$$

Writing $\theta_{i}:=i \mathbb{E} Z_{i}$ and $y_{i}:=\theta_{i}\left(1+E_{i}\right) /\left(i r_{i}\right)$, where $E_{i}:=\sum_{k=1}^{\infty} \varepsilon_{i k}$, we obtain

$$
\prod_{i=\lfloor x n\rfloor+1}^{n} \mathbb{P}\left[Z_{i}=0\right]=\exp \left(-\sum_{i=\lfloor x n\rfloor+1}^{n} \frac{\theta_{i}}{i}\right) \exp \left(-\sum_{i=\lfloor x n\rfloor+1}^{n} \frac{\theta_{i} E_{i}}{i}\right) \prod_{i=\lfloor x n\rfloor+1}^{n}\left(\frac{1-y_{i}}{e^{-y_{i}}}\right)^{r_{i}} .
$$

From Lemma 5.3 below,

$$
\exp \left(-\sum_{i=\lfloor x n\rfloor+1}^{n} \frac{\theta_{i}}{i}\right)=x^{\theta}\{1+O(\delta(m, \theta)+m /(n x))\}
$$

for any $m<\lfloor n x\rfloor / 2$; then, easily,

$$
\exp \left(-\sum_{i=\lfloor x n\rfloor+1}^{n} \frac{\theta_{i} E_{i}}{i}\right)=1+O\left(\mu_{\lfloor n x\rfloor}\right)
$$

and, because $y_{i} \leq 3 \theta_{\text {sup }} / 2 i \leq 1 / 2$ for all $i \geq i_{0}$,

$$
\prod_{i=\lfloor x n\rfloor+1}^{n}\left(\frac{1-y_{i}}{e^{-y_{i}}}\right)^{r_{i}}=1+O\left(n^{-1}\right),
$$

so that $\prod_{i=\lfloor x n\rfloor+1}^{n} \mathbb{P}\left[Z_{i}=0\right] \sim x^{\theta}$ under QLC. Combining this with 4.1) and (4.2), it follows that then

$$
\lim _{n \rightarrow \infty} \mathbb{P}\left[n^{-1} L^{(n)} \leq x\right]=\frac{x^{\theta} p_{\theta}(1 / x)}{x p_{\theta}(1)}=: F_{\theta}(x),
$$

where $F_{\theta}$ is a distribution function with density $f_{\theta}$ [ABT, p. 108]. If $\theta=1$, then $p_{\theta}(x)=e^{-\gamma} \rho(x)$. This proves the theorem.

Under QLC2, the convergence rate in (4.4) for each $x>0$ is of order $O\left(n^{-\eta_{3}}\right)$, for some $\eta_{3}>0$. 
One can also prove local versions of the convergence theorem. However, they have to involve the particular sequence $\theta_{i}$, since, for instance, $\mathbb{P}\left[L^{(n)}=r\right]=0$ if $\theta_{r}=0$, because then $Z_{r}$, and hence also $C_{r}^{(n)}$, are zero a.s. A typical result is as follows.

Theorem 4.2. If $Q L C$ holds, then, for any $0<x \leq 1$ such that $1 / x$ is not an integer, it follows that

$$
\lim _{n \rightarrow \infty}\left|n \mathbb{P}\left[L^{(n)}=\lfloor n x\rfloor\right]-\left(\theta_{\lfloor n x\rfloor} / \theta\right) f_{\theta}(x)\right|=0 .
$$

Under QLC2, the convergence rate is of order $O\left(n^{-} \eta_{4}\right)$, for some $\eta_{4}>0$.

Proof. Arguing as in the proof of the previous theorem,

$$
\mathbb{P}\left[L^{(n)}=\lfloor n x\rfloor\right]=\prod_{i=\lfloor n x\rfloor+1}^{n} \mathbb{P}\left[Z_{i}=0\right] \sum_{l=1}^{\lfloor n /\lfloor n x\rfloor\rfloor} \mathbb{P}\left[Z_{\lfloor n x\rfloor}=l\right] \frac{\mathbb{P}\left[T_{0,\lfloor n x\rfloor-1}=n-l\lfloor n x\rfloor\right]}{\mathbb{P}\left[T_{0, n}=n\right]} .
$$

Now, from Theorem 3.5 , the ratios

$$
\frac{\mathbb{P}\left[T_{0,\lfloor n x\rfloor-1}=n-l\lfloor n x\rfloor\right]}{\mathbb{P}\left[T_{0, n}=n\right]}
$$

are bounded as $n \rightarrow \infty$, uniformly for all $2 \leq l \leq\lfloor n /\lfloor n x\rfloor\rfloor$, provided that $1 / x$ is not an integer, so that $1-x\lfloor 1 / x\rfloor>0$. Then

$$
\begin{aligned}
\sum_{l \geq 2} \mathbb{P}\left[Z_{\lfloor n x\rfloor}=l\right] & \leq r_{\lfloor n x\rfloor} \mathbb{P}\left[Z_{\lfloor n x\rfloor, 1} \geq 2\right]+\left(\begin{array}{c}
r_{\lfloor n x\rfloor} \\
2
\end{array}\right)\left(\mathbb{P}\left[Z_{\lfloor n x\rfloor, 1}=1\right]\right)^{2} \\
& \leq \frac{1}{\lfloor n x\rfloor} \mu_{\lfloor n x\rfloor}+\frac{\left(\theta^{*}\right)^{2}}{2\lfloor n x\rfloor^{2}}
\end{aligned}
$$

implying that $\lim _{n \rightarrow \infty} n \sum_{l \geq 2} \mathbb{P}\left[Z_{\lfloor n x\rfloor}=l\right]=0$. Hence the sum of the terms for $l \geq 2$ on the right hand side of $(4.6)$ contributes an asymptotically negligible amount to the quantity $n \mathbb{P}\left[L^{(n)}=\lfloor n x\rfloor\right]$ as $n \rightarrow \infty$. For the $l=1$ term in $(4.6)$, both the product and the ratio of point probabilities are treated as in the proof of Theorem 4.1, giving the limit $x^{\theta-1} p_{\theta}((1-x) / x) / p_{\theta}(1)$, and

$$
\left|\mathbb{P}\left[Z_{\lfloor n x\rfloor}=1\right]-\theta_{\lfloor n x\rfloor} /\lfloor n x\rfloor\right| \leq \mu_{\lfloor n x\rfloor} /\lfloor n x\rfloor,
$$

so that

$$
\lim _{n \rightarrow \infty}\left|n \mathbb{P}\left[L^{(n)}=\lfloor n x\rfloor\right]-x^{\theta-1}\left\{p_{\theta}((1-x) / x) / p_{\theta}(1)\right\} x^{-1} \theta_{\lfloor n x\rfloor}\right|=0 .
$$

This completes the proof of (4.5). The remaining statement follows as usual, by taking greater care of the magnitudes of the errors in the various approximation steps.

In order to relax the condition that $1 / x$ is not integral, it is necessary to strengthen the assumptions a little; for example, if $x=1 / 2$ and $n$ is even, the contribution from the $l=2$ term in (4.6) is of order $O\left(\varepsilon_{n / 2,2} n^{1-\theta}\right)$, which could be large for $\theta<1$. In order to get a limit of $f_{\theta}(x)$ without involving the individual values $\theta_{i}$, it is necessary to average the point probabilities over an interval of integers around $\lfloor n x\rfloor$, of a length that grows with $n$, but is itself of magnitude $o(n)$. 


\subsection{The spectrum of small components}

We prove an analogue of the Kublius fundamental lemma (Kubilius, 1964) for quasi-logarithmic structures, and thus extend results of Arratia et al. (1995) and [ABT, Theorem 7.7]; see also the corresponding result of Manstavičius (2009), proved under different conditions.

Theorem 4.3. For $a / n \leq \alpha_{0}$, where $\alpha_{0}$ is small enough that $\min _{m \geq 1} \varepsilon_{5}^{\prime}(n, a, m) \leq \frac{1}{2} p_{\theta}(1)$, we have

$$
d_{\mathrm{TV}}\left(\mathscr{L}\left(C_{1}^{(n)}, \ldots, C_{a}^{(n)}\right), \mathscr{L}\left(Z_{1}, \ldots, Z_{a}\right)\right) \leq \varepsilon_{6}(n, a),
$$

where the order of magnitude of $\varepsilon_{6}(n, a)$ is given in (4.16) below.

If QLC holds, then

$$
\lim _{n \rightarrow \infty} d_{\mathrm{TV}}\left(\mathscr{L}\left(C_{1}^{(n)}, \ldots, C_{a_{n}}^{(n)}\right), \mathscr{L}\left(Z_{1}, \ldots, Z_{a_{n}}\right)\right)=0
$$

for every non-negative integer sequence $a_{n}=\mathrm{o}(n)$. If QLC2 holds, the convergence rate is of order $\{(a+1) / n\}^{\eta_{5}}$ for some $\eta_{5}>0$.

Proof. The proof is similar to that of [ABT, Theorem 5.2]. We fix an $n$ with the required properties, and we set $p_{k}:=\mathbb{P}\left[T_{a, n}=k\right]$. Then the conditioning relation entails

$$
\begin{aligned}
d_{\mathrm{TV}} & \left(\mathscr{L}\left(C_{1}^{(n)}, \ldots, C_{a}^{(n)}\right), \mathscr{L}\left(Z_{1}, \ldots, Z_{a}\right)\right) \\
= & \sum_{k=1}^{n} \mathbb{P}\left[T_{0, a}=k\right] \frac{\left(\mathbb{P}\left[T_{0, n}=n\right]-p_{n-k}\right)^{+}}{\mathbb{P}\left[T_{0, n}=n\right]} \\
\leq & \sum_{k=0}^{\lfloor n / 2\rfloor} \sum_{l=0}^{\lfloor n / 2\rfloor} \mathbb{P}\left[T_{0, a}=k\right] \mathbb{P}\left[T_{0, a}=l\right] \frac{\left(p_{n-l}-p_{n-k}\right)^{+}}{\mathbb{P}\left[T_{0, n}=n\right]} \\
& +\sum_{k=0}^{\lfloor n / 2\rfloor} \sum_{l=\lfloor n / 2\rfloor+1}^{n} \mathbb{P}\left[T_{0, a}=k\right] \mathbb{P}\left[T_{0, a}=l\right] \frac{p_{n-l}}{\mathbb{P}\left[T_{0, n}=n\right]}+\sum_{k=\lfloor n / 2\rfloor+1}^{n} \mathbb{P}\left[T_{0, a}=k\right] .
\end{aligned}
$$

We now separately bound the three terms in (4.8).

The first term is just

$$
\sum_{0 \leq k<l \leq n / 2} \mathbb{P}\left[T_{0, a}=k\right] \mathbb{P}\left[T_{0, a}=l\right] \frac{\left|p_{n-l}-p_{n-k}\right|}{\mathbb{P}\left[T_{0, n}=n\right]} .
$$

Now, from Theorem 3.5, using the bound given in (3.25), we have

$$
\left|n p_{n-r}-p_{\theta}(1-r / n)\right| \leq \varepsilon_{5}^{\prime}(n, a, m), \quad 0 \leq r \leq n / 2,
$$

so that, in (4.9),

$$
n\left|p_{n-l}-p_{n-k}\right| \leq\left|p_{\theta}(1-l / n)-p_{\theta}(1-k / n)\right|+2 \varepsilon_{5}^{\prime}(n, a, m) \leq c_{1}(\theta) n^{-1}|k-l|+2 \varepsilon_{5}^{\prime}(n, a, m),
$$

for any choice of $m$ and for some constant $c_{1}(\theta)$. Since also, from Theorem 3.5, $n \mathbb{P}\left[T_{0, n}=n\right]$ is uniformly bounded below whenever $\varepsilon_{5}^{\prime}(n, a, m) \leq \frac{1}{2} p_{\theta}(1)$, it follows that the first term in 4.8 is of order

$$
O\left(n^{-1} \mathbb{E} T_{0, a}+\min _{m \geq 1} \varepsilon_{5}^{\prime}(n, a, m)\right)=O\left(n^{-1} a+\min _{m \geq 1} \varepsilon_{5}^{\prime}(n, a, m)\right) .
$$


For the second term in (4.8), we have two bounds. First,

$$
\sum_{l=\lfloor n / 2\rfloor+1}^{n} \mathbb{P}\left[T_{0, a}=l\right] \frac{\mathbb{P}\left[T_{a, n}=n-l\right]}{\mathbb{P}\left[T_{0, n}=n\right]} \leq \frac{n \max _{n / 2 \leq l \leq n} \mathbb{P}\left[T_{0, a}=l\right]}{n \mathbb{P}\left[T_{0, n}=n\right]},
$$

where the denominator is uniformly bounded below whenever $\varepsilon_{5}^{\prime}(n, a, m) \leq \frac{1}{2} p_{\theta}(1)$, and, for $n / 2 \leq$ $l \leq n$ and $a \leq n / 4$,

$n \mathbb{P}\left[T_{0, a}=l\right] \leq 2 l \mathbb{P}\left[T_{0, a}=l\right] \leq 2 \theta^{*} \mathbb{P}\left[T_{0, a} \geq n / 4\right]+2 \varepsilon_{2}(a, 0, m) \leq 8\left(\theta^{*}\right)^{2}(a / n)+2 \varepsilon_{2}(a, 0, m)$,

from (3.15), with the last step following because $\mathbb{E} T_{0, a} \leq a \theta^{*}$. The second bound is given by

$$
\begin{aligned}
\sum_{l=\lfloor n / 2\rfloor+1}^{n} \mathbb{P}\left[T_{0, a}=l\right] \frac{\mathbb{P}\left[T_{a, n}=n-l\right]}{\mathbb{P}\left[T_{0, n}=n\right]} & \leq \mathbb{P}\left[T_{0, a} \geq n / 2\right] \frac{\sup _{j \in \mathbb{Z}_{+}} \mathbb{P}\left[T_{a, n}=j\right]}{\mathbb{P}\left[T_{0, n}=n\right]} \\
& \leq \frac{2 a \theta^{*} D^{1}\left(T_{a, n}\right)}{n \mathbb{P}\left[T_{0, n}=n\right]}
\end{aligned}
$$

again using Markov's inequality, and the asymptotically important part is $a D^{1}\left(T_{a, n}\right)$. Thus the second term in (4.8) is of order

$$
O\left(n^{-1} a+\min \left\{\min _{m \geq 1} \varepsilon_{2}(a, 0, m), a D^{1}\left(T_{a, n}\right)\right\}\right) .
$$

Finally, the third term in (4.8) can be simply bounded from above by

$$
2 n^{-1} \mathbb{E} T_{0, a} \leq 2 \theta^{*} n^{-1} a .
$$

Combining (4.11), (4.14) and (4.15) proves the first part of the theorem, with

$$
\varepsilon_{6}(n, a)=O\left(n^{-1} a+\min _{m \geq 1} \varepsilon_{5}^{\prime}(n, a, m)+\min \left\{\min _{m \geq 1} \varepsilon_{2}(a, 0, m), a D^{1}\left(T_{a, n}\right)\right\}\right) .
$$

The remaining statements follow as usual.

\subsection{Additive arithmetic semigroups}

We now return to the example given in the Introduction, of a quasi-logarithmic combinatorial structure that is not logarithmic. In Knopfmacher's (1979) additive arithmetic semigroups, the elements of norm $n$ can be decomposed into prime elements, with $C_{i}^{(n)}$ the number of prime elements of norm $i$ in the decomposition of a randomly chosen element of norm $n$. The joint distribution of $\left(C_{1}^{(n)}, \ldots, C_{n}^{(n)}\right)$ satisfies the conditioning relation, with $Z_{i} \sim \operatorname{NB}\left(p(i), q^{-i}\right)$, so that

$$
\mathbb{P}\left[Z_{i}=k\right]:=\left(\begin{array}{c}
p(i)+k-1 \\
k
\end{array}\right) q^{-i k}\left(1-q^{-i}\right)^{n}, \quad k \in \mathbb{Z}_{+},
$$

with the convention that $Z_{i}=0$ if $p(i)=0$. Here, $p(i)$ denotes the total number of prime elements of norm $i$, and $q>1$ enters through the assumption that the number $g(n)$ of elements of size $n$ satisfies

$$
g(n)=q^{n} \sum_{j=1}^{r} c_{j} n^{\rho_{j}-1}+\mathrm{O}\left(q^{n} n^{-\gamma}\right)
$$


for real numbers $\rho_{1}<\cdots<\rho_{r}$ and $c_{1}, \ldots, c_{r}$, with $\rho_{r}>0, c_{r}>0$, and with $\gamma>1$, an analogue of a condition under which Beurling (1937) examined prime number theorems of so called generalized integers. In particular, Zhang (1996, Theorem 6.2) shows, under condition (4.17) with $\gamma>2$, that $\theta_{i}:=i \mathbb{E} Z_{i}=i p(i) q^{-i} /\left(1-q^{-i}\right)=\theta_{i}^{\prime}+o(1)$, where $\left\{\theta_{i}^{\prime}\right\}_{i \in \mathbb{N}}$ is the integer skeleton of a sinusoidal mixture function

$$
\theta_{t}^{\prime}:=\theta+\sum_{l=1}^{L} \lambda_{l} \cos \left(2 \pi f_{l} t-\varphi_{l}\right), \quad t \in \mathbb{R},
$$

with $\theta:=\rho_{r}>0$, amplitudes $\lambda_{l}>0$ such that $\sum_{l=1}^{L} \lambda_{l} \leq \theta$, non-integral frequencies $f_{l} \in[0, \infty) \backslash \mathbb{Z}_{+}$ and phases $0 \leq \varphi_{l}<2 \pi$; note that his arguments and notation are non-probabilistic. In many examples, the sum of cosines is empty $(L=0)$, and the structure logarithmic. When this is the case, the asymptotic behaviour of the small and large components is as described in the Introduction: see Arratia et al. (2005) for these and other results. Here, we are interested in establishing asymptotics in the case when $L \geq 1$.

First, note that the same sequence $\theta_{i}^{\prime}$, for integral $i$, is obtained, if each $f_{l}$ is replaced by its fractional part $f_{l}-\left\lfloor f_{l}\right\rfloor$, so that the values of $f_{l}$ can be taken to lie in $(0,1)$; and then that, if $f_{l}>1 / 2$, it can first be replaced by $f_{l}-1$, and then by $1-f_{l}$ if also $\varphi_{l}$ is replaced by $-\varphi_{l}$, again without changing the $\theta_{i}^{\prime}$. Hence we may assume that $f_{l} \in(0,1 / 2]$ for all $l$.

Clearly, for $L \geq 1$, the sequence $\theta_{i}^{\prime}$ is in general not convergent in the usual sense, but, in view of the properties of trigonometric functions,

$$
\sum_{t=i_{1}+1}^{i_{2}} \cos \left(2 \pi f_{l} t-\varphi_{l}\right) \leq \frac{1}{\sin \pi f_{l}},
$$

whatever the values of $i_{1}, i_{2}$, so that $\delta(m, \theta)=O\left(m^{-1}\right) \rightarrow 0$ as $m \rightarrow \infty$; and $\mu_{i}=O\left(q^{-i}\right)$ as $i \rightarrow \infty$. Hence the condition QLC2 is satisfied by these structures if, for some $r, s$ coprime, $k \in \mathbb{N}$ and $\psi>0$, the set $\left\{i: \min \left(\theta_{i}, \theta_{i+d}\right) \geq \psi\right\}$ has at least one element in each $k$-interval $\{j k+1, \ldots,(j+1) k\}$ for all $d \in D:=\{r\} \cup\left\{s 2^{g}, g \in \mathbb{Z}_{+}\right\}$, for all $j$ sufficiently large: see the comment following Theorem 2.1. Now, if $\sum_{l=1}^{L} \lambda_{l}<\theta$, all the $\theta_{i}^{\prime}$ are uniformly bounded below by $\psi_{1}:=\theta-\sum_{l=1}^{L} \lambda_{l}>0$, and the condition QLC2 is clearly satisfied with any choice of $r, s$ and $k$ if $\psi=\frac{1}{2} \psi_{1}$. If $\sum_{l=1}^{L} \lambda_{l}=\theta$, define

$$
V_{l}(i):=\min _{n \in \mathbb{Z}}\left|2 \pi f_{l} i-\varphi_{l}-(2 n+1) \pi\right|,
$$

and suppose that

$$
V_{l}(i) \leq \delta_{l}:=\pi \min \left\{f_{l}, 1-2 f_{l}\right\}
$$

for some pair $l, i$. Then, if $n_{*}:=n_{*}(l, i)$ attains the minimum in 4.19), it is immediate that

$$
2 \pi f_{l}(i+r)-\varphi_{l}-\left(2 n_{*}+1\right) \pi \geq \pi f_{l} \geq \delta_{l}
$$

for all $r \geq 1$. On the other hand,

$$
\begin{aligned}
\left(2\left(n_{*}+1\right)+1\right) \pi-\left(2 \pi f_{l}(i+r)-\varphi_{l}\right) & \geq 2 \pi-2 r \pi f_{l}-\pi\left(1-2 f_{l}\right) \\
& \geq \pi\left(1-2 f_{l}\right) \geq \delta_{l},
\end{aligned}
$$

if $r \leq 2$. Hence, if $V_{l}(i) \leq \delta_{l}$, then $V_{l}(i+r) \geq \delta_{l}$ for $r=1$, 2. Setting

$$
L_{i}:=\left\{1 \leq l \leq L: V_{l}(i) \geq \delta_{l}\right\}
$$


this implies that, for each $i$,

$$
L_{i}^{c} \subset L_{i+1} \cap L_{i+2} \text {. }
$$

Now it follows from the inequality $1-\cos x \geq c x^{2}$ in $|x| \leq \pi / 3$, with $2 c=1-\pi^{2} / 108$, that

$$
\theta_{i}^{\prime} \geq c \sum_{l \in L_{i}} \lambda_{l} \delta_{l}^{2}
$$

Define $\psi_{2}:=\frac{c}{2} \sum_{l=1}^{L} \lambda_{l} \delta_{l}^{2}$. Then if, for some $i, \theta_{i}^{\prime} \leq \psi_{2}$, we have $c \sum_{l \notin L_{i}} \lambda_{l} \delta_{l}^{2} \geq \psi_{2}$, and it follows from the above considerations that

$$
\theta_{i+r}^{\prime} \geq c \sum_{l \notin L_{i}} \lambda_{l} \delta_{l}^{2} \geq \psi_{2}, \quad r=1,2,
$$

in view of (4.20) and (4.21). Thus every interval of length $k=3$ far enough from the origin contains an index $i$ with $\min \left(\theta_{i}, \theta_{i+d}\right) \geq \frac{1}{2} \psi_{2}$, whatever the value of $d$. Hence the condition QLC2 is satisfied for any choice of $r, s$ co-prime, provided that $\psi_{2}>0$.

There remains the possibility that $f_{l}=1 / 2$ for all $1 \leq l \leq L$, in which case $\psi_{2}=0$. If any of the $\varphi_{l}$ are not multiples of $\pi$, the function $\theta_{i}^{\prime}$ is once again uniformly bounded away from 0 , and the same is true if one is an even multiple of $\pi$ and another an odd one. Hence there are only two cases in which QLC2 is not satisfied:

$$
\theta_{t}^{\prime}:=\theta\{1+\cos (\pi t)\} \quad \text { and } \quad \theta_{t}^{\prime}:=\theta\{1+\cos (\pi(t-1))\}
$$

In the former case, $\theta_{i}^{\prime}>0$ only for even $i$, and if $\mathbb{P}\left[Z_{i}=0\right]=1$ for all odd $i$ then $d_{\mathrm{TV}}\left(\mathscr{L}\left(T_{0, n}\right), \mathscr{L}\left(T_{0, n}+1\right)\right)=1$ for all $n$; hence, for instance, Theorem 3.5 cannot be expected to be true. In the latter case, we can take $D:=\left\{2^{g+1}, g \geq 0\right\}$, and use Theorem 2.2 to show that $d_{\mathrm{TV}}\left(\mathscr{L}\left(T_{a, n}\right), \mathscr{L}\left(T_{a, n}+1\right)\right)=O\left(\{(a+1) / n\}^{\eta}\right)$ for some $\eta>0$; the rest of the argument is then as before.

\section{Technical bounds}

Here we collect some technical results that are needed to smooth out the irregularities in the sequence of $\theta_{i}$ 's. Let $\theta>0$, and let $\left\{\theta_{i}\right\}_{i \in \mathbb{N}}$ be any non-negative sequence. Let $\theta_{*}:=\sup _{i \in \mathbb{N}} \theta_{i}$ and $\theta_{*}^{\prime}:=\theta \vee \theta_{*}$. For every $m, n \in \mathbb{N}$ we set

$$
\delta(m, \theta):=\sup _{j \geq 0}\left|\frac{1}{m} \sum_{i=1}^{m} \theta_{j m+i}-\theta\right| .
$$

Lemma 5.1. Let $\left\{y_{i}\right\}_{i \in \mathbb{N}}$ be a real-valued sequence, and $0 \leq l<n$. Then

$$
\left|\sum_{i=l+1}^{n}\left(\theta_{i}-\theta\right) y_{i}\right| \leq\left\{\begin{array}{c}
\left(2 m \theta_{*}^{\prime}+n \delta(m, \theta)\right)\|y\|+\theta_{*}(n m / 8)\|\Delta y\| \\
2 m \theta_{*}^{\prime}\|y\|+\delta(m, \theta) \sum_{i=1}^{n}\left|y_{i}\right| \\
+m^{-1} \theta_{*} \sum_{l=1}^{m} \sum_{l^{\prime}=1}^{m} \sum_{j=1}^{\lfloor n / m\rfloor-1}\left|y_{j m+l}-y_{j m+l^{\prime}}\right|
\end{array}\right.
$$

where $\|y\|:=\max _{l<i \leq n}\left|y_{i}\right|$ and $\|\Delta y\|:=\max _{l<i<n}\left|y_{i+1}-y_{i}\right|$. 
Proof. For any $0 \leq j \leq\lfloor n / m\rfloor$, we have

$$
\begin{aligned}
\left|\sum_{i=j m+1}^{(j+1) m}\left(\theta_{i}-\theta\right) y_{i}\right| & =\left|\sum_{i=j m+1}^{(j+1) m}\left\{\left(\bar{\theta}^{(j)}-\theta\right) y_{i}+\left(\theta_{i}-\bar{\theta}^{(j)}\right)\left(y_{i}-\bar{y}^{(j)}\right)\right\}\right| \\
& \leq\left|\theta-\bar{\theta}^{(j)}\right| \sum_{i=j m+1}^{(j+1) m}\left|y_{i}\right|+\theta_{*} \sum_{i=j m+1}^{(j+1) m}\left|y_{i}-\bar{y}^{(j)}\right|,
\end{aligned}
$$

where $\bar{\theta}^{(j)}:=m^{-1} \sum_{i=j m+1}^{(j+1) m} \theta_{i}$ and $\bar{y}^{(j)}:=m^{-1} \sum_{i=j m+1}^{(j+1) m} y_{i}$. Note also that

$$
\sum_{i=j m+1}^{(j+1) m}\left|y_{i}-\bar{y}^{(j)}\right| \leq\left\{\begin{array}{l}
\left(m^{2} / 8\right)\|\Delta y\| ; \\
m^{-1} \sum_{l=1}^{m} \sum_{l^{\prime}=1}^{m}\left|y_{j m+l}-y_{j m+l^{\prime}}\right|
\end{array}\right.
$$

The lemma now follows by bounding the sum $\sum_{i=l+1}^{n}\left(\theta_{i}-\theta\right) y_{i}$ in $m$-blocks.

Lemma 5.2. Let $T$ be an $\mathbb{Z}_{+}$-valued random variable, and $0 \leq l<n, m \geq 1$.

(i) For every bounded function $g: \mathbb{Z}_{+} \rightarrow \mathbb{R}$, we have

$$
\left|\sum_{i=l+1}^{n}\left(\theta_{i}-\theta\right) \mathbb{E} g(T+i)\right| \leq\|g\|\left(2 \theta_{*}^{\prime} m+n \delta(m, \theta)+\frac{1}{4} \theta_{*} m n d_{\mathrm{TV}}(\mathscr{L}(T), \mathscr{L}(T+1))\right) .
$$

(ii) For every $k \in \mathbb{N}$, we have

$$
\left|\sum_{i=l+1}^{n}\left(\theta_{i}-\theta\right) \mathbb{P}[T+i=k]\right| \leq \delta(m, \theta)+m\left(2 \theta_{*}^{\prime}+\frac{1}{6} \theta_{*} m\right) d_{\mathrm{TV}}(\mathscr{L}(T), \mathscr{L}(T+1)) .
$$

Proof. (i) We apply the first inequality in Lemma 5.1, with $y_{i}:=\mathbb{E} g(T+i)$, noting that $\|\Delta y\| \leq$ $2 d_{\mathrm{TV}}(\mathscr{L}(T), \mathscr{L}(T+1))$.

(ii) We apply the second inequality in Lemma 5.1, with $y_{i}:=\mathbb{P}[X+i=k]$, and observe that then

$$
\sup _{j \in \mathbb{Z}_{+}} \mathbb{P}[T=j] \leq d_{\mathrm{TV}}(\mathscr{L}(T), \mathscr{L}(T+1)),
$$

as for (3.14), and that

$$
\sum_{j=1}^{\lfloor n / m\rfloor-1}\left|y_{j m+l}-y_{j m+l^{\prime}}\right| \leq\left|l-l^{\prime}\right| d_{\mathrm{TV}}(\mathscr{L}(T), \mathscr{L}(T+1)) .
$$

Lemma 5.3. If $0<2 m \leq l \leq n$, then

$$
\exp \left|\sum_{i=l+1}^{n} \frac{\theta_{i}-\theta}{i}\right| \leq \exp \left\{2 m\left(1+\theta_{*}\right) / l\right\}\left(\frac{n}{l}\right)^{\delta(m, \theta)} \leq e^{1+\theta_{*}}\left(\frac{n}{l}\right)^{\delta(m, \theta)} .
$$


Proof. Choosing $y_{i}:=1 / i$, the second inequality in Lemma 5.1 gives

$$
\begin{aligned}
\left|\sum_{i=l+1}^{n} \frac{\theta_{i}-\theta}{i}\right| & \leq 2 \frac{m}{l}+\delta(m, \theta) \sum_{i=l+1}^{n} \frac{1}{i}+\theta_{*} \sum_{j=\lfloor l / m\rfloor}^{\lfloor n / m\rfloor-1} \frac{1}{j(j+1)} \\
& \leq 2\left(1+\theta_{*}\right) \frac{m}{l}+\delta(m, \theta) \sum_{i=l+1}^{n} \frac{1}{i},
\end{aligned}
$$

and the lemma follows.

The authors wish to thank the referees for a number of helpful suggestions.

\section{References}

Arratia, R., A. D. Barbour, and S. Tavaré. 2003. Logarithmic combinatorial structures: a probabilistic approach, EMS Monographs in Mathematics, European Mathematical Society (EMS), Zürich. MR2032426

Arratia, R., A. D. Barbour, and S. Tavaré. 2005. A probabilistic approach to analytic arithmetic on algebraic function fields, Math. Proc. Cambridge Philos. Soc. 139, no. 1, 1-26.

Arratia, R., D. Stark, and S. Tavaré. 1995. Total variation asymptotics for Poisson process approximations of logarithmic combinatorial assemblies, Ann. Probab. 23, no. 3, 1347-1388.

Barbour, A. D., L. H. Y. Chen, and W.-L. Loh. 1992. Compound Poisson approximation for nonnegative random variables via Stein's method, Ann. Probab. 20, no. 4, 1843-1866.

Barbour, A. D. and B. L. Granovsky. 2005. Random combinatorial structures: the convergent case, J. Combin. Theory Ser. A 109 , no. 2, 203-220.

Beurling, A. 1937. Analyse de la loi asymptotique de la distribution des nombres premiers généralisés, Acta Math. 68, 255291.

Chung, F. and L. Lu. 2006. Concentration inequalities and martingale inequalities: a survey, Internet Mathematics 3, 79127.

Dickman, K. 1930. On the frequency of numbers containing prime factors of a certain relative magnitude, Ark. Math. Astr. Fys. 22, no. 10, 1-14.

Freiman, G. A. and B. L. Granovsky. 2002. Asymptotic formula for a partition function of reversible coagulationfragmentation processes, Israel J. Math. 130, 259-279.

Kingman, J. F. C. 1977. The population structure associated with the Ewens sampling formula, Theoret. Population Biology 11, no. 2, 274-283.

Knopfmacher, J. 1979. Analytic arithmetic of algebraic function fields, Lecture Notes in Pure and Applied Mathematics, vol. 50, Marcel Dekker Inc., New York.

Kubilius, J. 1964. Probabilistic methods in the theory of numbers, Translations of Mathematical Monographs, Vol. 11, American Mathematical Society, Providence, R.I.

Lindvall, T. 2002. Lectures on the coupling method, Dover Publications Inc., Mineola, NY.

Manstavičius, E. 2009. Strong convergence on weakly logarithmic combinatorial assemblies, arXiv:0903.1051.

Mattner, L. and B. Roos. 2007. A shorter proof of Kanter's Bessel function concentration bound, Prob. Theory Rel. Fields 139, 191-205.

Mineka, J. 1973. A criterion for tail events for sums of independent random variables, Z. Wahrscheinlichkeitstheorie und Verw. Gebiete 25, 163-170.

Rösler, U. 1977. Das 0-1-Gesetz der terminalen $\sigma$-Algebra bei Harrisirrfahrten, Z. Wahrscheinlichkeitstheorie und Verw. Gebiete 37, no. 3, 227-242. 
Rogers, L.C.G. 1999. Fastest coupling of random walks, J. London Math. Soc. 60, 630-640.

Vervaat, W. 1972. Success epochs in Bernoulli trials with applications in number theory, Mathematical Centre Tracts, vol. 42, Mathematisch Centrum, Amsterdam.

Zhang, W.-B. 1996. The prime element theorem in additive arithmetic semigroups. I, Illinois J. Math. 40, no. 2, 245-280. 